\title{
MONOTONICITY OF QUADRATURE APPROXIMATIONS
}

\author{
D. J. NEWMAN ${ }^{1}$
}

\begin{abstract}
For the general quadrature rules of Newton-Cotes we show that the convergence is monotone for the appropriate class of functions. Included are the well-known trapezoidal and Simpson rules.
\end{abstract}

Some time ago Szegö and Turán [3] showed that the Riemann sums $n^{-1} \sum_{i=1}^{n} f(i / n)$ form a nonincreasing sequence for any $f(x)$ which is increasing and concave. Recently Rivlin and I found that the trapezoidal sums $(f(0)+f(1)) / 2 n+n^{-1} \sum_{i=1}^{n-1} f(i / n)$ also form a nonincreasing sequence, this time for $f(x)$ satisfying $f^{\prime \prime}(x) \geqq 0, f^{\prime \prime \prime}(x) \leqq 0, f \in C^{3}$. An analogous result was obtained by Molluzo [2] for the "Simpson rule" where the appropriate condition turned out to be $f^{\mathrm{iv}}(x) \geqq 0, f^{\mathrm{v}}(x) \leqq 0, f \in C^{5}$.

In each case the theme seems to be that the proper signum for the first two derivatives that "count" is sufficient for monotonicity. Thus in the trapezoidal case, since the rule is exact for linear functions, the first derivatives' size does not count. Similarly Simpson's rule is exact for cubics and so there the first three derivatives do not count. [Molluzo also set up the machinery to verify the monotonicity for the so-called $\frac{3}{8}$-rule but the computations became unwieldy.]

The purpose of this note is to prove that this behavior does indeed persist for all of the Newton-Cotes rules. For reasons of brevity we will say "up" and "down" respectively to mean nondecreasing and nonincreasing and also we will say "plus" and "minus" to mean nonnegative and nonpositive.

LEMMA 1. Let $\mu(x)$ be a normalized $B V$ function on $[0,1]$ with $\mu(0)=0$ and let $\alpha \geqq 0$. The necessary and sufficient condition (nasc) that

$$
n^{-\alpha} \int_{0}^{1} F\left(\frac{x}{n}\right)+F\left(\frac{x+1}{n}\right)+F\left(\frac{x+n-1}{n}\right) d \mu(x)
$$

be a down sequence for every plus, down function, $F(x)$, is that both $x^{-\alpha} \mu(x)$ and $\mu(x)-\alpha x \mu(1)$ be don'n on $[0,1]$

Received by the editors October 2, 1972 and, in revised form, February 20, 1973.

AMS (MOS) subject classifications (1970). Primary 65D30, 65B99.

Key words and phrases. Monotonicity, quadrature.

${ }^{1}$ Supported in part by AFOSR-72-2380.

(c) American Mathematical Society 1974 
Proof. If we extend $\mu(x)$ to the whole positive line by the relation $\mu(x+1)=\mu(x)+\mu(1)$ then we have identically,

$$
\int_{0}^{1} F\left(\frac{x}{n}\right)+F\left(\frac{x+1}{n}\right)+\cdots+F\left(\frac{x+n-1}{n}\right) d \mu(x)=\int_{0}^{1} F(x) d \mu(n x) .
$$

Integrating by parts we find this is, in turn, equal to

$$
\mu(n) F(1)-\int_{0}^{1} \mu(n x) d F(x) .
$$

We desire therefore, that

$$
n^{-\alpha} \mu(n) F(1)-\int_{0}^{1}(n x)^{-\alpha} \mu(n x) x^{\alpha} d F(x)
$$

be a down sequence for every plus, down $F(x)$ and an obviously sufficient condition is that for each fixed $x \in(0,1],(n x)^{-\alpha} \mu(n x)$ be a down sequence. Furthermore choosing $F(x)$ as a simple jump function shows this condition to also be a necessary one.

Next we observe that if $t^{-\alpha} \mu(t)$ were a down function on $(0, \infty)$ this would surely insure the down nature of the sequence $(n x)^{-\alpha} \mu(n x)$. Conversely by choosing a small $x$ and large values of $n$ we can show that monotonicity of the sequences implies monotonicity of the function.

Thus far, then, our nasc is simply that $t^{-\alpha} \mu(t)$ be down on $(0, \infty)$ and we proceed to interpret this as a condition on $[0,1]$.

For $K$ any plus integer we have then that $(x+K)^{-\alpha}[\mu(x)+K \mu(1)]$ is down in $[0,1]$. We may differentiate this expression and obtain, in the language of measures, that

or

$$
(x+K)^{-\alpha} d \mu(x)-\alpha(x+K)^{-\alpha-1}[\mu(x)+K \mu(1)] d x \leqq 0
$$

$$
(x d \mu(x)-\alpha \mu(x) d x)+K(d \mu(x)-\alpha \mu(1) d x) \leqq 0 .
$$

This holding for all plus integers $K$, finally, is equivalent to both

$$
x d \mu(x)-\alpha \mu(x) d x \leqq 0, \quad d \mu(x)-\alpha \mu(1) d x \leqq 0
$$

and our proof is complete.

An important case for us will be that of "symmetric" $\mu(x)$, i.e., those for which $\mu(1-x)=\mu(1)-\mu(x)$. In that case the second of our conditions is a consequence of the first. Namely if $x d \mu(x) \leqq \alpha \mu(x) d x$ then

$$
\begin{aligned}
(1-x) d \mu(x) & =-(1-x) d \mu(1-x) \leqq \alpha \mu(1-x) d x \\
& =\alpha \mu(1) d x-\alpha \mu(x) d x
\end{aligned}
$$

and adding gives $d \mu(x) \leqq \alpha \mu(1) d x$. Thus we have the 
COROLlaRY. For symmetric $\mu(x)$ (with $\mu(0)=0$ ) the nasc that

$$
n^{-\alpha} \int_{0}^{1} F\left(\frac{x}{n}\right)+\cdots+F\left(\frac{x+n-1}{n}\right) d \mu(x)
$$

be down for all plus, down $F(x)$ is that $x^{-\alpha} \mu(x)$ be dow'n on $(0,1]$.

We now introduce the Newton-Cotes rules. So let $N$ be a positive odd integer. For $f(x)$ any continuous function on $[0,1]$ we denote by $A(x)$ the $N$ th degree polynomial interpolating $f(x)$ at $0,1 / N, 2 / N, \cdots, N / N$ and $B(x)$ the $(N-1)$ th degree polynomial interpolating $f(x)$ at $0,1 /(N-1)$, $2 /(N-1), \cdots,(N-1) /(N-1)$. The Newton-Cotes functionals of first and second kinds are given respectively by

$$
L^{(1)}(f(x))=\int_{0}^{1} A(x) d x, \quad L^{(2)}(f(x))=\int_{0}^{1} B(x) d x
$$

and the Newton-Cotes rules are then given by

$$
\begin{gathered}
L_{n}(f(x))=\frac{1}{n} L\left(f\left(\frac{x}{n}\right)+f\left(\frac{x+1}{n}\right)+\cdots+f\left(\frac{x+n-1}{n}\right)\right), \\
L=L^{(1)}, \quad L^{(2)} .
\end{gathered}
$$

For example the trapezoidal rule familiarly written as

$$
\frac{f(0)+2 f(1 / n)+2 f(2 / n)+\cdots+f(1)}{2 n}
$$

emerges as $L_{n}^{(1)}(f(x))$ for $N=1$. Similarly Simpson's rule

$$
\frac{f(0)+4 f(1 / 2 n)+2 f(2 / 2 n)+\cdots+4 f((2 n-1) / 2 n)+f(1)}{6 n}
$$

is simply $L_{n}^{(2)}(f(x))$ when $N=3$.

In this context we state our general

THEOREM. If $f(x) \in C^{N+2}, f^{(N+1)}(x) \geqq 0, f^{(N+2)}(x) \leqq 0^{2}$ on $[0,1]$ then $L_{n}(f(x))$ is a down sequence $\left(L=L^{(1)}\right.$ or $\left.L^{(2)}\right)$.

Proof. It is well known that for $f(x)$ a polynomial of degree $N$ the rules are exact, i.e., that $L^{(1)}(f(x))=L^{(2)}(f(x))=\int_{0}^{1} f(x) d x$. This says that $\int_{0}^{1} A(x) d x=\int_{0}^{1} f(x) d x$ and $\int_{0}^{1} B(x) d x=\int_{0}^{1} f(x) d x$ and the first of these is trivial since $A(x) \equiv f(x)$. The second relation follows from the observations that $f(x)-B(x)$ is a constant multiple of

$$
\prod(x)=\prod_{j=0}^{N-1}(x-j /(N-1))
$$

and $\int_{0}^{1} \Pi(x) d x=0$ since $\Pi(1-x) \equiv-\Pi(x)$.

\footnotetext{
${ }^{2}$ Sending $x$ into $1-x$ allows us to replace these conditions, if we wish, by $f^{(N+1)}(x) \geqq 0, f^{(N+2)}(x) \geqq 0$.
} 
Thus $L(f(x))-\int_{0}^{1} f(x) d x$ annihilates all polynomials of degree $N$ and as such is a (bounded) linear functional of $f^{(N+1)}(x)$ alone. Write $F(x)=f^{(N+1)}(x)$ and conclude that $L(f(x))-\int_{0}^{1} f(x) d x=\int_{0}^{1} F(x) d \mu(x)$ so that

$$
\begin{aligned}
L_{n}(f(x)) & -\int_{0}^{1} f(x) d x \\
& =n^{-(N+2)} \int_{0}^{1} F\left(\frac{x}{n}\right)+F\left(\frac{x+1}{n}\right)+\cdots+F\left(\frac{x+n-1}{n}\right) d \mu(x)
\end{aligned}
$$

and we find ourselves in the exact situation described by our Lemma. Indeed it is a simple observation that our $\mu(x)$ is symmetric and we may even avail ourselves of the Corollary to that Lemma. To prove our theorem, then, we need only check that $t^{-(N+2)} \mu(t)$ is down on $[0,1]$ and we prove in fact that $t^{-(N+1)} \mu(t)$ is.

We now derive a formula for $\mu(t)$ implicit in the standard results on the Newton-Cotes rules (see [1]). The approach we use, however, is self-contained and avoids lengthy discussion in the calculus of finite differences.

For convenience in the forthcoming calculation let us expand the underlying interval from $[0,1]$ and redefine

$$
L^{(1)}(f(x))=\int_{0}^{N} A(x) d x=\int_{0}^{N} f^{(N+1)}(x) d \mu^{(1)}(x)+\int_{0}^{N} f(x) d x
$$

(where $A(x)$ is the interpolating polynomial to $f(x)$ at $0,1,2, \cdots, N$ ):

$$
L^{(2)}(f(x))=\int_{0}^{N-1} B(x) d x=\int_{0}^{N-1} f^{(N+1)}(x) d \mu^{(2)}(x)+\int_{0}^{N-1} f(x) d x
$$

(where $B(x)$ is the interpolating polynomial to $f(x)$ at $0,1,2, \cdots, N-1$ ).

In this new normalization we now write $d v(x)=d \mu^{(1)}(x)-d \mu^{(2)}(x)$ and we will prove the monotonicity of $x^{-(N+1)} \mu^{(2)}(x)$ and $x^{-(N+1)} v(x)$ which is more than sufficient. If indeed we note that $A(x)-B(x)$ is a constant multiple of $x(x-1) \cdots(x-N+1)$ then we obtain

$$
\int_{0}^{N-1}(A(x)-B(x)) d x=C \int_{0}^{N-1} x(x-1) \cdots(x-N+1) d x=0
$$

and we conclude that

so that

$$
L^{(1)}(f(x))-L^{(2)}(f(x))=\int_{N-1}^{N} A(x) d x
$$

$$
\int_{0}^{N} f^{(N+1)}(x) d v(x)=\int_{N-1}^{N}(A(x)-f(x)) d x
$$


and of course we also have

$$
\int_{0}^{N-1} f^{(N+1)}(x) d \mu(x)=\int_{0}^{N-1}(B(x)-f(x)) d x .
$$

Now set $f(x)=e^{-\lambda x}$. By Lagrange's interpolation formula we have

$$
\begin{aligned}
& A(x)=\frac{x(x-1) \cdots(x-N)}{\lfloor N} \sum_{j=0}^{N}(-1)^{j}\left(\begin{array}{c}
N \\
j
\end{array}\right) \frac{e^{-j \lambda}}{j-x}, \\
& B(x)=\frac{-x(x-1) \cdots(x-N+1)}{\lfloor N-1} \sum_{j=0}^{N-1}(-1)^{j}\left(\begin{array}{c}
N-1 \\
j
\end{array}\right) \frac{e^{-j \lambda}}{j-x},
\end{aligned}
$$

also, setting $\lambda=0$, e.g., we obtain

$$
\begin{aligned}
& 1=\frac{x(x-1) \cdots(x-N)}{\lfloor N} \sum_{j=0}^{N}(-1)^{j}\left(\begin{array}{c}
N \\
j
\end{array}\right) \frac{1}{j-x}, \\
& 1=-\frac{x(x-1) \cdots(x-N+1)}{\lfloor N-1} \sum_{j=0}^{N-1}(-1)^{j}\left(\begin{array}{c}
N-1 \\
j
\end{array}\right) \frac{1}{j-x},
\end{aligned}
$$

so that, by subtraction,

$$
\begin{aligned}
& A(x)-f(x)=\frac{x(x-1) \cdots(x-N)}{\lfloor N} \sum_{j=0}^{N}(-1)^{j}\left(\begin{array}{c}
N \\
j
\end{array}\right) \frac{e^{-j \lambda}-e^{-x \lambda}}{j-x} \\
& B(x)-f(x)=-\frac{x(x-1) \cdots(x-N+1)}{\lfloor N-1} \sum_{j=0}^{N-1}(-1)^{j}\left(\begin{array}{c}
N-1 \\
j
\end{array}\right) \frac{e^{-j \lambda}-e^{-x \lambda}}{j-x} .
\end{aligned}
$$

Next note that $\left(e^{-\lambda j}-e^{-\lambda x}\right) /(j-x)=\lambda \int_{0}^{1} e^{-\lambda x s} e^{-\lambda j(1-s)} d s$ so that the above formulas become

(3) $A(x)-f(x)=\frac{-x(x-1) \cdots(x-N)}{\lfloor N} \lambda \int_{0}^{1} e^{-\lambda x s}\left(1-e^{-\lambda(1-s)}\right)^{N} d s$,

(4) $B(x)-f(x)=\frac{x(x-1) \cdots(x-N+1)}{\underline{\mid N-1}} \lambda \int_{0}^{1} e^{-\lambda x s}\left(1-e^{-\lambda(1-s)}\right)^{N-1} d s$.

We now abbreviate these by writing

$$
\begin{aligned}
P(x) & =-\frac{x(x-1) \cdots(x-N)}{\lfloor N}, \\
Q^{\prime}(x) & =\frac{x(x-1) \cdots(x-N+1)}{\lfloor-1}, \quad Q(0)=0
\end{aligned}
$$

and we observe the trivial facts that $P(x) \geqq 0$ in $[N-1, N]$ and $Q(N-1)=0$, and also the somewhat less trivial fact that $Q(x) \geqq 0$ on $[0, N-1]$. A 
simple proof can be given by induction. Since $(t-N+2)(t-N+1)$ is plus, down in $[0, N-3]$ we have, for $x$ in this interval,

$\int_{0}^{x} t(t-1) \cdots(t-N+1) d t=(N-1)(N-2) \int_{0}^{\xi} t(t-1) \cdots(t-N+3) d t$

and the latter is $\geqq 0$ by the inductive hypothesis. For $x \in[N-3, N-1]$, on the other hand, we simply use $Q(N-1-x)=Q(x)$.

Comparing (3) and (4) then with (1) and (2) gives

$$
\begin{gathered}
\int_{0}^{N} e^{-\lambda x} d v(x)=\int_{N-1}^{N} P(x) \int_{0}^{1} e^{-\lambda x s}\left(\frac{1-e^{-i(1-s)}}{\lambda}\right)^{N} d t d x, \\
\int_{0}^{N-1} e^{-\lambda x} d \mu(x)=\int_{0}^{N-1} Q(x) \int_{0}^{1} e^{-i x s}\left(\frac{1-e^{-i(1-s)}}{\lambda}\right)^{N-1} s d s d x,
\end{gathered}
$$

where in the latter we have integrated by parts.

If we now write $\left(1-e^{-\lambda(1-s)}\right) / \lambda=\int_{0}^{1-s} e^{-i u} d u$ so that

$$
\left(\frac{1-e^{-\lambda(1-s)}}{\lambda}\right)^{K}=\int_{0}^{1-s} \cdots \int_{0}^{1-s} \exp \left\{-\lambda\left(u_{1}+u_{2}+\cdots+u_{k}\right)\right\} d u_{1} \cdots d u_{k},
$$

we can replace (6) and (7) by

$$
\begin{aligned}
& \int_{0}^{N} e^{-\lambda x} d v(x) \\
& \quad=\int_{N-1}^{N} P(x) \int \cdots \int_{\mathscr{R}} e^{-\lambda\left(x s+u_{1}+u_{2}+\cdots+u_{N^{\prime}}\right)} d u_{1} d u_{2} \cdots d u_{N} d s d x
\end{aligned}
$$

where $\mathscr{R}$ is defined by $u_{i} \geqq 0, s \geqq 0, u_{i}+s \leqq 1$,

$$
\begin{array}{rl}
\int_{0}^{N-1} e^{-\lambda x} & d \mu(x) \\
= & \int_{0}^{N-1} Q(x) \int \cdots \int_{\mathscr{R}^{\prime}} e^{-\lambda\left(x s+u_{1}+\cdots+u_{s-1}\right)} d u_{1} \cdots d u_{N-1} d s d x .
\end{array}
$$

We can now identify $v(t)$ and $\mu(t)$ since these equalities hold for all $\lambda$. We have namely

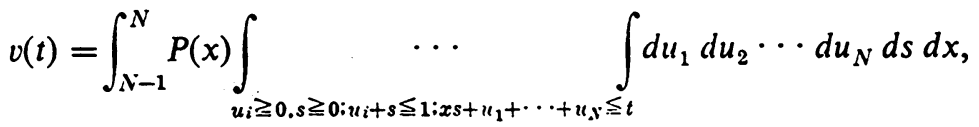

$$
\begin{aligned}
& \mu(t)=\int_{0}^{N-1} Q(x) \int_{u_{i} \geq 0, s \geq 0: u_{i}+s \leqslant 1: x+u_{1}+\cdots+u_{s-1} \leqq t} \int_{\leqq} s d u_{1} \cdots d u_{N-1} d s d x
\end{aligned}
$$


changing variables by $u_{i}=t U_{i}, s=t S$ we obtain finally

$$
\begin{aligned}
\frac{v(t)}{t^{N+1}} & =\int_{N-1}^{N} P(x) \int_{U_{i} \geqq 0 . S \geqq 0: U i+S \leq 1 / t: x S+U_{1}+\cdots+V_{N} \leq 1} \\
\frac{\mu(t)}{t^{N+1}}= & \int_{0}^{N-1} Q(x) \\
& \times \int_{U_{i} \geqq 0 . S \geqq 0: U_{i+S} \leqq 1 / t: x S+U_{1}+\cdots+U_{N-1} \leqq 1} d U_{1} \cdots d U_{N} d S d x,
\end{aligned}
$$

The inner integrals are clearly down as functions of $t$ and so the proof is complete.

Since we had the luck to prove that $t^{-(N+1)} \mu(t)$ is down, rather than only that $t^{-(N+2)} \mu(t)$ is, it seems we should be able to conclude more monotonicity than that of $L_{n}$. Indeed this is the case and in fact we find that we have proved

THEOREM'. If $f(x) \in C^{N+2}, f^{(N+1)}(x) \geqq 0, f^{(N+2)}(x) \leqq 0$ on $[0,1]$ then $n L_{n}(f(x))-n \int_{0}^{1} f(x) d x$ is doun $\left(L=L^{(1)}\right.$ or $\left.L^{(2)}\right)$.

In particular applying this to the trapezoidal rule gives the interesting

Corollary. For $f(x) \in C^{3}, f^{\prime \prime}(x) \geqq 0, f^{\prime \prime \prime} \leqq 0$ on $[0,1]$ we have

$$
\sum_{j=1}^{n} f\left(\frac{j}{n+1}\right)-\sum_{j=1}^{n-1} f\left(\frac{j}{n}\right) \leqq \int_{0}^{1} f(x) d x .
$$

\section{REFERENCES}

1. L. M. Milne-Thompson, Calculus of finite differences, Macmillan, New York, 1933.

2. J. Molluzo, Doctoral Thesis, Yeshiva University, 1972 (unpublished).

3. G. Szegö and P. Turán, On the monotone convergence of certain Riemann sums, Publ. Math. Debrecen 8 (1961), 326-335. MR 25 \#1267.

Department of Mathematics, Belfer Graduate School of Science. Yeshiva University, NEW YORK, NEW YORK 10033 